\title{
SCIDoc

\section{Successful Management of a Patient with Florid Diabetic Retinopathy}

\author{
Case Report
}

Ahmad SS*, Ghani SA, Alexander SM

Department of Ophthalmology, Queen Elizabeth Hospital, Kota Kinabalu, Malaysia.

\section{Abstract}

Florid diabetic retinopathy is an unusual, aggressive type of diabetic retinopathy. It is seen in young patients with poor metabolic control. Despite advances in treatment the overall prognosis of this condition remains poor. A 21-year old female patient was seen by us and found to have florid diabetic retinopathy despite an adequate metabolic control by oral hypoglycemic agents. The patient underwent multiple laser and orbital floor triamcinolone acetonide injections. Later the left eye developed persistent vitreous hemorrhage for which a vitrectomy with silicone oil injection was done. During a 4 year follow-up she has shown a visually and anatomical stable response.

Keywords: Diabetic Retinopathy; Vitreous Hemorrhage; Light Coagulation; Vitrectomy.

\section{Introduction}

Florid diabetic retinopathy (FDR) is a term usually applied to a particularly aggressive, bilateral proliferative diabetic retinopathy. It is a difficult condition to manage, with the patient often ending up with blindness over a course of time despite treatment. Here in, we report a young patient with FDR who refused intra-vitreal injections, but was successfully managed by an appropriate utilization of laser photocoagulation, orbital floor steroid injections and vitreo-retinal surgery.

\section{Materials and Methods}

7 years back a 21-year old unmarried, non-obese female patient was referred to us for diabetic retinopathy screening. She was on oral metformin (100mg once daily) and oral gliclazide (80mg twice daily). Her blood glucose level was consistently maintained at 72-90mg/dl. No systemic abnormalities, including nephropathy, were detected.

The patient had no ocular symptoms and her best corrected visual acuity was $6 / 18$ in both eyes. The anterior segments in both eyes were normal. However, fundus examination showed profuse florid diabetic retinopathy, with "coralliform" vessels branching out from the optic disc vessels. Areas of neovascularisation else where (NVE) were also visible. Clinically significant macular edema (CSME) was prominently present in both eyes (Figure 1, 2).

The patient underwent multiple sessions of grid and panretinal photocoagulation, augmented initially with orbital floor triamcinolone acetonide injection, as the patient refused any intravitreal injections. After a period of time, the condition dampened appreciably. The FDR and CSME regressed and the BCVA improved to $6 / 10$ in both eyes. After a period of nearly 1 year the patient started to develop vitreous hemorrhage in the left eye. She was advised for a vitrectomy which she continued to refuse. Ultimately, nearly 3 years after being seen here she underwent a vitrectomy with endolaser and silicon oil injection. Subsequently, the condition resolved appreciably with a small area of residual fibrosis remaining and the patient regaining good vision which is maintained over 4 years of follow-up. On the last visit, 3 months back her BCVA was $6 / 9$ in the right eye and $6 / 15$ in the left eye (Figure 3-6).

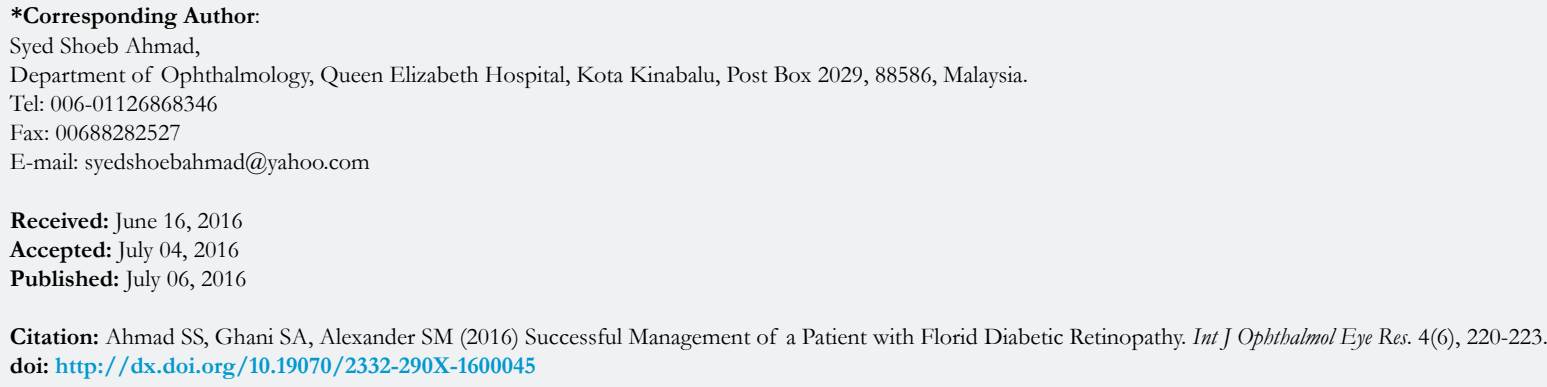

Copyright: Ahmad SS ${ }^{\odot}$ 2016. This is an open-access article distributed under the terms of the Creative Commons Attribution License, which permits unrestricted use, distribution and reproduction in any medium, provided the original author and source are credited. 
Figure 1. Right eye of the patient on initial presentation.

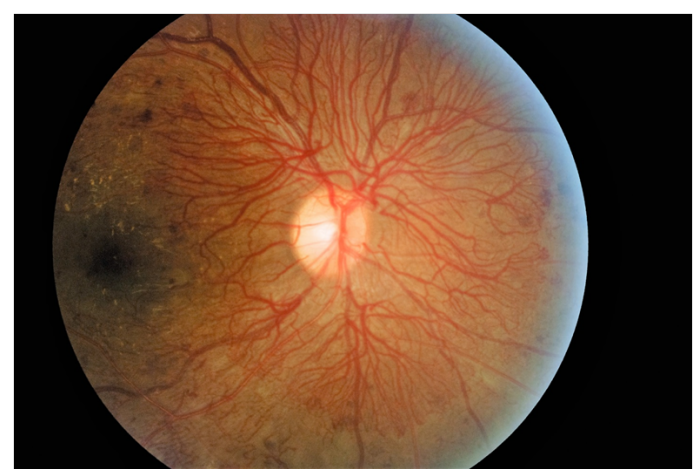

Figure 2. Left eye of the patient on initial presentation.

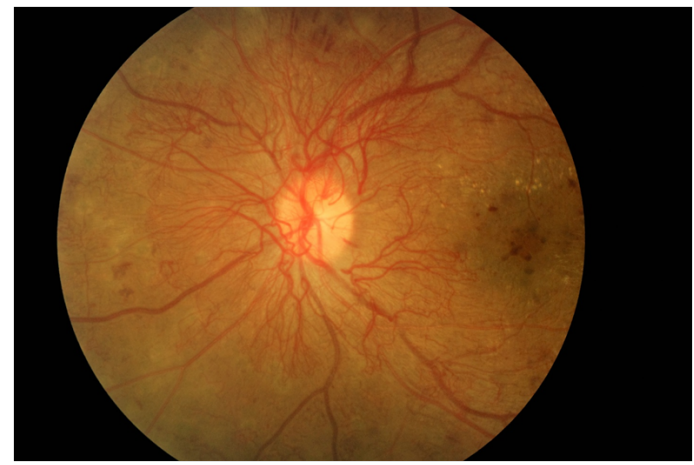

Figure 3. Right eye of patient after initial treatment.

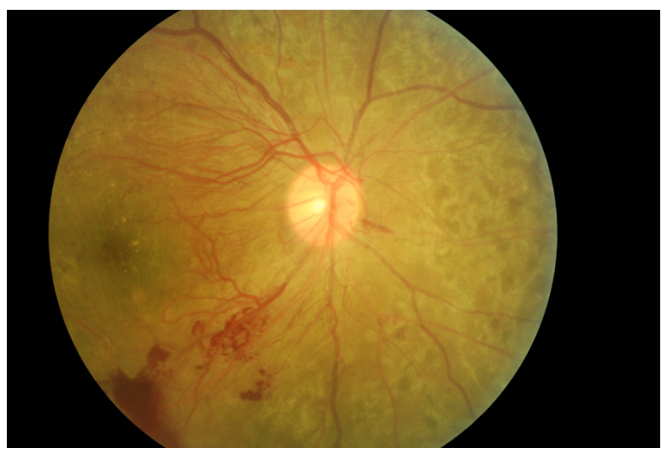

Figure 4. Left eye of patient after initial treatment.

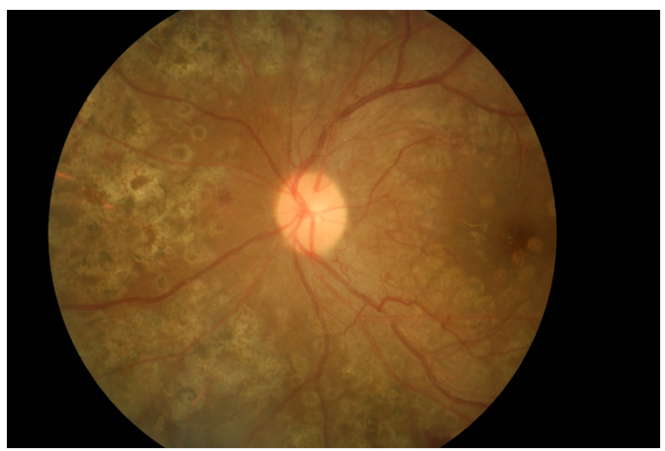

\section{Results and Discussions}

Florid diabetic retinopathy (FDR) is a term used by Kohner and Oakley to describe a severe, bilateral, rapidly progressive type of proliferative diabetic retinopathy occurring in young adults [1]. FDR is characterized by its occurrence in Type I diabetic patients who are less than 40 years of age and who show a progression to proliferative diabetic retinopathy in less than 6 months [2].
Beaumont and Hallows have described FDR as a "rapid, bleeding and blinding" condition [3]. The pathogenesis of FDR is likely due to diffuse retinal ischemia with breakdown of the blood-retinalbarrier in widespread areas of the retina. Kitano has shown that acute hypoglycemic episodes along with transient increases in the serum levels of insulin-like growth factor 1 (IGF-1) and vitreous levels of vascular endothelial growth factor (VEGF) contribute to the development of FDR [4]. 
Figure 5. Right eye of patient on last review.

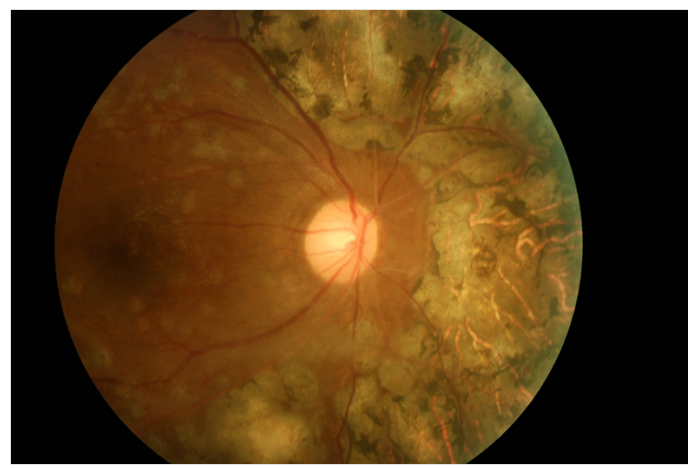

Figuer 6. Left eye of patient following vitrectomy.

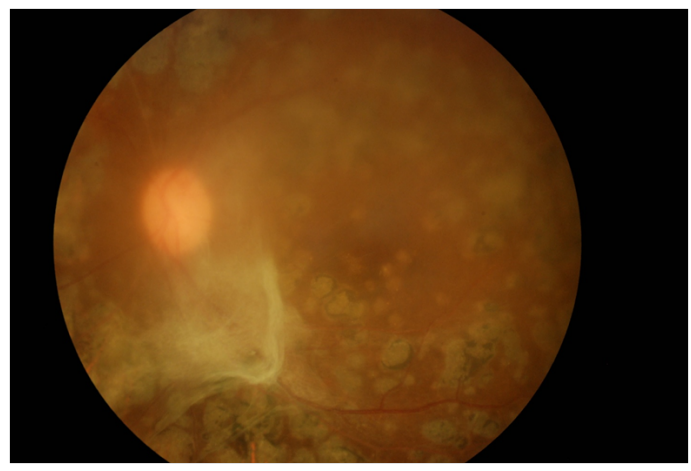

Poor long-term metabolic control of diabetes is found to be a significant finding in patients with FDR. The HbA1c levels should be monitored and kept within normal target range [5]. FDR is also reported to regress with the usage of an insulin-infusion device [6] and also with continuous subcutaneous insulin infusion [7]. Curiously, in our patient however, the metabolic control was persistently good. Associated hypertension in patients having FDR is another risk factor [8].

FDR is characterized by a rapid progression from moderate non-proliferative diabetic retinopathy to florid, aggressive neovascularisation over the disc and elsewhere. Devastating complications can develop over a period of weeks, underlining the importance of frequent, close follow-up of the patients. [8]. Major complications which can lead to blindness include: Tractional retinal detachment, neovascular glaucoma and anterior hyaloidal fibrovascular proliferation (AHFP) [2, 9]. In a study of FDR, Lattanzio et al., divided patients into two groups depending on their baseline retinal condition and feasibility of photocoagulation: group 1 included eyes which were amenable to laser photocoagulation and if required, subsequent vitreoretinal surgery; whereas group 2 consisted of eyes with more advanced diabetic retinopathy, requiring primary vitreo retinal surgery and laser photocoagulation. After a follow-up of 54 months, $75 \%$ of eyes in group 1 were found to have a favorable anatomical outcome with a resultant mean final visual acuity of 0.47 . In group 2, diabetic retinopathy progressed in $68 \%$ of eyes and a mean final visual acuity of 0.14 could be achieved. The latter group also had 6 times higher rates of blindness as compared to group 1 (31\% versus 5\%). The authors suggested that early diagnosis and treatment can halt the progression of FDR in three out of four cases [8].
In a 17-year old girl with FDR and Donahue syndrome, Casati et al., achieved satisfactory regression of NVD and maintenance of good visual acuity following PRP [10]. However, Seyer-Hansen reported complete blindness in a 22 -year old patient within 30 months of diagnosis of diabetes mellitus [11]. If left untreated, FDR can lead to blindness over a period of 2-4 years [5]. In our patient, satisfactory vision was achieved by judicious use of medical and surgical techniques to manage the condition.

According to Agardh, PRP should be instituted early, once the stage of severe non-proliferative diabetic retinopathy is detected [5]. However, vitreo-retinal surgery might be required in cases having tractional RD involving or threatening the macula, combined tractional and rhegmatogenous $\mathrm{RD}$, rapid onset of fibrovascular proliferations (FVP) despite PRP with or without extramacular tractional $\mathrm{RD}$, vitreous hemorrhage without spontaneous clearing, or recurrent vitreous hemorrhage [2]. In these cases, preoperative intravitreal injections of anti-VEGF agents have been found to be particularly beneficial. These agents facilitate the dissection of proliferative fibrovascular membranes, reduce intraoperative bleeding as well as postoperative AFVP [2].

\section{Conclusion}

While FDR is a condition fraught with a grave prognosis, yet it is possible to achieve a favorable response by the utilization of grid/focal and pan-retinal photocoagulation, orbital floor steroid injections and vitreo-retinal surgery.

\section{References}

[1]. Kohner EM, Oakley NW (1975) Diabetic retinopathy. Metabolism 24(9): 1085-1102.

[2]. Couturier A, Dupas B, Guyomard JL, Massin P (2014) Surgical outcomes 
of florid diabetic retinopathy treated with antivascular endothelial growth factor. Retina 34(10): 1952-1959.

[3]. Beaumont P, Hallows FC (1972) Classification of diabetic retinopathy with therapeutic implications. Lancet 299(7747): 419-424.

[4]. Kitano S, Funatsu H, Tanaka Y, Suzuk M (2005) Vitreous levels of IGF-1 and VEGF in florid diabetic retinopathy. Invest Ophthalmol Vis Sci 46: 347.

[5]. Agardh E (2010) Rapid, bloody, and blinding diabetic retinopathy. Acta Ophthalmol 88(3): 274-278.

[6]. Irsigler K, Kirtz H, Najemnik C, Freyler H (1980) Regression of florid proliferative diabetic retinopathy through achievement of normoglycaemic over five months by means of a portable insulin-dosage apparatus. Wien Klin Wochenschr 92(8): 270-276.

[7]. White MC, Kohner EM, Pickup JC, Keen H (1981) Reversal of diabetic retinopathy by continuous subcutaneous insulin infusion: a case report. $\mathrm{Br} \mathrm{J}$ Ophthalmol 65(5): 307-311.
[8]. Lattanzio R, Brancato R, Bandello FM, Azzolini C, Malegori A, et al., (2001) Florid diabetic retinopathy (FDR): a long-term follow-up study. Graefes Arch Clin Exp Ophthalmol 239(3): 182-187.

[9]. Favard C, Guyot-Argenton C, Assouline M, Marie-Lescure C, Pouliquen YJ (1996) Full panretinal photocoagulation and early vitrectomy improve prognosis of florid diabetic retinopathy. Ophthalmology 103(4): 561-574.

[10]. Casati S, Zoppini G, Muggeo M, Marchini G (2010) Sustained regression of florid diabetic retinopathy in a patient with Donahue syndrome (leprechaunism). Eur J Ophthalmol 20(1): 224-227.

[11]. Seyer-Hansen K, Faurschou S (1985) Severe proliferative retinopathy in a young man with diabetes of very short duration. Acta Med Scand 217(5): 571-574. 